\title{
Das späte Frühgeborene: eine Herausforderung in der Stillberatung
}

Hella R. Köster, Hebamme, Dipl.-Medizinpädagogin, IBCLC, Solingen

Späte Frühgeborene adaptieren sich meistens recht gut nach der Geburt und haben überwiegend ein ausreichend hohes Geburtsgewicht, um auf der regulären Wochenstation betreut werden zu können. Das täuscht allerdings darüber hinweg, dass sie noch eine nennenswerte organische und neurologische Unreife aufweisen und über unzureichende Energiereserven verfügen. Eine Folge ist ineffektives Stillen mit dem Risiko für mehr oder weniger schwerwiegende Komplikationen. Was ist bei der Stillberatung besonders zu beachten, um bei diesen Kindern effektives Stillen zu ermöglichen?

\section{Das späte Frühgeborene}

Das späte Frühgeborene (late preterm infant, LPI) ist international definiert als ein Frühgeborenes mit einem Gestationsalter zwischen $34+0$ und $36+6$ Wochen post menstruationem.

Die Anzahl der Frühgeburten in Deutschland beträgt insgesamt ca. $9 \%$, davon sind etwa 2/3 späte Frühgeborene, was den Zahlen in anderen entwickelten Ländern entspricht. Ihr Anteil an den Geburten hat in den letzten Jahren zugenommen, wie auch das Bewusstsein, dass es sich bei dieser Gruppe um Risikokinder handelt. Dies wurde inzwischen mehrfach durch internationale Studien untermauert. Eine Konsequenz daraus ist, dass die geplante Sectio nach neueren Empfehlungen vorzugsweise erst mit frühestens $38+0$ bzw. erst mit 39+0 SSW durchgeführt wird [1].

\section{Risikofaktor Unreife}

Im Prinzip sind bei späten Frühgeborenen alle Risikofaktoren der Unreife gegeben, mit einer abnehmenden Tendenz bei steigendem Gestationsalter. Andererseits wurde festgestellt, dass die entsprechenden Risiken teilweise auch noch mit $37+0$ bis $38+6$ Wochen erhöht sind.

\section{Merkmale der Unreife bei späten Frühgeborenen}

- Schwierigkeiten, die Körpertemperatur zu halten

- Erhöhte Infektionsanfälligkeit

- Verzögerte Bilirubinausscheidung

- Instabile Atmung

- Neurologische Unreife

- Trinkschwäche
Zahlen einer Studie in der Schweiz von 2012 verdeutlichen, wie viel häufiger Komplikationen bei späten Frühgeborenen im Vergleich zu reifen Neugeborenen sind (Tab. 1). Das Risiko, mindestens eine Komplikation zu entwickeln, ist bei ihnen demnach 7,6-mal so hoch wie bei reifen Neugeborenen [2].

Als Komplikation kommen auch schwere Atemstörungen, die eine künstliche Beatmung oder ein CPAP erforderlich machen, gehäuft vor. Ebenso ist die Sterberate verglichen mit reifen Neugeborenen erhöht und ein schlechter neurologischer Zustand zum Ende des Aufenthaltes in

Tab. 1 Komplikationen bei späten Frühgeborenen und reifen Neugeborenen [2].

\begin{tabular}{|l|l|l|}
\hline & $\begin{array}{l}\text { Späte Frühgeborene } \\
(\mathbf{n}=\mathbf{5 5 0})\end{array}$ & $\begin{array}{l}\text { Reife Neugeborene } \\
(\mathbf{n}=\mathbf{1 6 8 6})\end{array}$ \\
\hline Hyperbilirubinämie & $47,7 \%$ & $3,4 \%$ \\
\hline Atemstörungen & $34,7 \%$ & $4,6 \%$ \\
\hline Hypoglykämie & $14,3 \%$ & $0,6 \%$ \\
\hline Fütterungsprobleme & $8,3 \%$ & $0,6 \%$ \\
\hline Hypothermie & $2,5 \%$ & $0,6 \%$ \\
\hline Klinikaufenthalt post partum & 9,9 Tage & 5,6 Tage \\
\hline
\end{tabular}


der Neonatologie ist häufiger [3]. Mögliche Langzeitfolgen sind geistiger Entwicklungsrückstand mit Lernschwierigkeiten und niedrigem Schulabschluss, psychomotorischer Entwicklungsrückstand und Verhaltensauffälligkeiten [1, 4].

\section{Risikofaktor Stillen}

Paradoxerweise sind späte Frühgeborene besonders dann für die genannten Komplikationen gefährdet, wenn sie gestillt werden $[5,6,7]$. Dabei sind gerade das Stillen bzw. die Muttermilchernährung dafür bekannt, die Defizite, welche eine Frühgeburt mit sich bringen, optimal auszugleichen.

Der Grund ist vielmehr die Problemkonstellation aus dem Unvermögen des Kindes ausreichend effektiv zu stillen und einem unzureichend angepassten Still- und Muttermilchmanagement.

Damit gehen ein erhöhter initialer Gewichtsverlust, eine langsame Gewichtszunahme bis hin zur schweren Gedeihstörung mit Dehydratation einher. Dies wirkt sich bei dem bereits vorhandenen Mangel an Energiereserven umso gravierender aus und ist der Ausgangspunkt für die meisten Komplikationen.

\section{Ein klassisches Beispiel}

Sara war mit 35 SSW und einem Gewicht von $2720 \mathrm{~g}$ geboren worden. Ihrer Mutter Anna wurde mitgeteilt, dass sie reif sei, wegen ihres guten Gewichts, und sie wurde früh entlassen, weil sie zudem einen gesunden Eindruck machte. Sara ging gut an die Brust, ermüdete aber sehr schnell beim Stillen. 3 Tage später wurde sie wegen hoher Bilirubinwerte und Gewichtsverlust wieder stationär aufgenommen. Die Milchbildung bei Anna war zurückgegangen und sie wurde angewiesen, mit der Fütterung von Formulanahrung zu beginnen [8].
Die Hyperbilirubinämie ist der häufigste Grund für eine Rehospitalisierung, gefolgt von Dehydratation bzw. Mangelernährung und (Verdacht auf) Sepsis [7].

Insgesamt haben späte Frühgeborene, die bei der Klinikentlassung nach der Geburt ausschließlich gestillt wurden, ein 1,5-2 mal so hohes Risiko für eine Rehospitalisierung wie nicht gestillte späte Frühgeborene und gestillte reife Neugeborene [5].

\section{Die Stillproblematik beim späten Frühgeborenen}

Mehrere Studien der letzten Jahre zu Stillhäufigkeit und -dauer bei späten Frühgeborenen zeigen ein einheitliches Bild $[5,7,9,10]$. Danach werden späte Frühgeborene

- deutlich seltener gestillt [5],

- seltener innerhalb der 1 . Stunde nach der Geburt gestillt,

- meistens kürzer gestillt (häufig nur wenige Wochen),

- und seltener ausschließlich gestillt als reife Neugeborene.

Obgleich bei ihnen dieselben Risikofaktoren für das Nicht-Stillen wie bei reifen Neugeborenen gefunden werden bezüglich Rauchen der Mutter, Ehestatus und Bildung, konnte die Unreife als ein davon unabhängiger Risikofaktor festgemacht werden [11].

\section{Neurologische Entwicklung}

Es wird davon ausgegangen, dass das unzureichende Stillverhalten zu einem wesentlichen Teil an der neurologischen Unreife dieser Kinder liegt [6, 7]. So zeigt die neurologische Entwicklung im Mutterleib, dass $1 / 3$ des Hirnwachstums erst in den letzten 6-8 SSW erfolgt [6]. Dementsprechend liegt das Gewicht des Gehirns mit 34 SSW bei $65 \%$ und mit 36 SSW erst bei $80 \%$ des reifen Gehirns [12]. Hinzu kommt eine weitere Ausdifferenzierung der Hirnstrukturen in diesem Zeitraum, z.B. der Hirnwindungen.
Auf der anderen Seite ist bekannt, dass gerade das Stillen bzw. die Muttermilchernährung maßgeblich zu einer besseren Hirnentwicklung beiträgt als Ersatznahrung [13]. Ebenso wird dadurch die psychomotorische Entwicklung der vorbelasteten Frühgeborenen bestmöglich unterstützt [20]. Der Stillvorgang an sich beugt zudem späteren Sprech- und Essstörungen vor [21].

\section{Stillprobleme bei späten Frühgeborenen}

Wodurch ist das unreife, unzureichende Stillverhalten beim späten Frühgeborenen im Einzelnen charakterisiert? (Siehe Merkmale des unreifen Stillverhaltens.)

Die Saugschwäche wird davon bestimmt, dass der Saugdruck und der Expressionsdruck gering sind. Der Saugdruck entwickelt sich dabei langsamer als der Expressionsdruck [7]. Er ist notwendig, um die Brustwarze zu formen und den Saugschluss herzustellen und zu halten. Das Saugen an einem Flaschensauger ist deshalb für späte Frühgeborene zunächst einfacher, wodurch sie nicht in dem Maße von Komplikationen betroffen sind wie gestillte Kinder. Die Koordination von Saugen-Atmen-Schlucken reift erst nach 36 Wochen p.m. aus. Bis $\mathrm{zu} \leq 35$ Wochen tritt noch gehäuft apnöisches Schlucken $(\geq 3 \times$ Schlucken ohne Atmung) auf und auch danach gibt es noch eine hohe Variabilität an Schluck-Atem-Mustern [14]. Diese Faktoren können das Stillen zusätzlich erschweren.

In Abhängigkeit von ihrem Temperament wirken manche späte Frühgeborene zunächst kräftig und lebhaft, sodass ihnen weniger Beachtung geschenkt wird [15], im Sinne von „die schaffen das schon“. Bei genauerer Beobachtung des Stillverhaltens wird dann aber deutlich, dass auch sie die typischen Stillschwierigkeiten eines späten Frühgeborenen haben. Auffallend sind dann ausgedehnte Schlafphasen von 4 Std. und mehr, nachdem sie sich zuvor verausgabt haben. 


\section{Merkmale des unreifen Stillverhaltens:}

- Die Kinder sind schläfriger, werden seltener wach und haben kürzere Wachphasen.

- Sie zeigen nur subtile Stillzeichen.

- Beim Stillen haben sie weniger Ausdauer und Durchhaltevermögen.

- Sie haben mehr Probleme, die Brust zu erfassen und zu formen.

- Sie verlieren die Brust leicht in einer Saugpause.

- Sie zeigen eine Saugschwäche, d.h. die Kraftentwicklung beim Stillen ist geringer.

- Sie haben schwächere Reflexe.

- Sie haben Probleme, Saugen, Schlucken und Atmen zu koordinieren.

- Ihnen fehlt die notwendige Energie für den Stillvorgang.

- Sie können keine stabile Stillposition einnehmen (niedriger Muskeltonus).

- Evtl. ungünstiges Verhältnis zwischen Größe der Brustwarze und kindlichem Mund.

Meistens wird das ausgeprägte Schlafbedürfnis ihres späten Frühgeborenen von den Eltern als Zeichen dafür gesehen, dass es satt und zufrieden ist. Auch gehen sie davon aus, dass ein hungriges Kind sich von selber melden wird. So ist es manchmal schwer zu vermitteln, dass ein spätes Frühgeborenes der besonderen Aufmerksamkeit bedarf und sie sich nicht auf ein ganz „normales“ Verhalten verlassen können. Diese Unsicherheit ist für Eltern manchmal nur schwer auszuhalten [16].

\section{Milchbildung}

Ein zusätzlicher Aspekt der Stillproblematik beim späten Frühgeborenen ist ein mangelhafter Aufbau der Milchbildung. Die Laktogenese II (hormonelle gesteuerte Milchbildung nach Wegfall der Plazentahormone, Milcheinschuss) setzt bei ihren Müttern häufig verspätet ein, weil bei ihnen meistens ein oder mehrere Risikofaktoren dafür vorliegen wie:

- Hoher Body-Mass-Index

- Diabetes

- SS-induzierte Hypertonie

- Vorzeitige Wehentätigkeit

- Längere Bettruhe

- Sectio caesarea

- Geburtskomplikationen

- Medikamente, einschließlich Magnesiumsulfat u. a. antepartum/intrapartum Medikation [7].
Besonders kritisch ist der Übergang zur Laktogenese III (volle Milchbildung), die maßgeblich von der effektiven Leerung der Brust abhängt, nach dem Prinzip von Angebot und Nachfrage. Bereits während des Übergangs zur Laktogenese II werden die Strukturen der Brustdrüse durch effektives Stillen auf die volle Milchbildung vorbereitet. Findet keine adäquate Leerung und Stimulation der Brust statt, kommt es stattdessen sogar zur Downregulierung der Milchmenge und die Milchbildungskapazität ist dauerhaft eingeschränkt. Das kann zu einem späteren Zeitpunkt durch ein intensiviertes Still- oder Pumpmanagement, wenn überhaupt, nur mit viel Mühe aufgeholt werden [7].

Eine unzureichende Milchbildung macht sich häufig erst nach der Klinikentlassung bemerkbar. Während das späte Frühgeborene bei einer guten Laktogenese II an einer leicht fließenden Brust möglicherweise noch ausreichend Milch erhält, baut sich die Milchmenge danach nicht entsprechend den steigenden Anforderungen auf [17]. Es kommt zur Stagnation der Gewichtszunahme, zur zunehmend eingeschränkten Vitalität und schließlich zur Gedeihstörung beim Kind. Das Zufüttern von Muttermilchersatznahrung wird dann meistens unumgänglich.
So ist es bei späten Frühgeborenen in der Regel unverzichtbar, bis zu einem reifen Saugverhalten zusätzlich zum Stillen abzupumpen, um eine ausreichende Milchbildung sicherzustellen.

\section{Stillmanagement}

\section{Kritische Aspekte}

Das späte Frühgeborene wird in den meisten Fällen vom Kreißsaal auf die reguläre Wochenstation übernommen und dort nach denselben Standards versorgt wie ein reifes Neugeborenes. Das gilt auch für das Stillmanagement und die Stillberatung, d.h. es fehlen spezifische Standards für diese Kinder.

Außerdem lassen sich nicht nur die Eltern, sondern auch das Gesundheitspersonal durch ein gutes Geburtsgewicht leicht über die Unreife des Kindes hinwegtäuschen. So wiegt ein Neugeborenes, das mit 40 SSW ein Gewicht von 3800 g erreicht hätte, mit 36 SSW schon 3000 g. Ein weiterer kritischer Aspekt ist ausschließliches Stillen bei einer frühen Klinikentlassung, ohne dass ein zusätzliches Pumpmanagement geregelt ist.

Die psycho-soziale Belastung der Mutter und mangelndes Selbstvertrauen bei einem unreifen Kind beeinträchtigen häufig die Motivation, das Stillen und den Milchaufbau unter den erschwerten Bedingungen auf sich zu nehmen [11, 16, 18]. Eine Trennung von Mutter und Kind, z.B. bei Verlegung des Kindes auf die Neugeborenenintensivstation, wirkt sich besonders nachteilig auf das Stillen aus [11]. Bei Zwillingen unter den späten Frühgeborenen und auch bei sehr kleinen Frühgeborenen scheint das Stillen hingegen besser unterstützt zu werden. Möglicherweise werden sie stärker als eine Risikogruppe für Stillschwierigkeiten wahrgenommen [11]. 


\section{Assessment}

Die wichtigste Voraussetzung für ein angemessenes Stillmanagement ist die gezielte Erfassung des kindlichen Zustandes und seines Stillverhaltens. Beim späten Frühgeborenen sollte allein schon das bei der Geburt rechnerisch ermittelte Schwangerschaftsalter von $<38$ SSW Anlass zu einer frühzeitigen und engmaschigen Kontrolle geben. Dazu gehören:

- Einschätzung der tatsächlichen Reife und des Ernährungszustandes

(Frage nach Hypotrophie)

- Beurteilung von Anlegen, Saugvermögen und Milchtransfer:

- frühzeitige professionelle Einschätzung, d.h. innerhalb von 12 bis 24 Std. p.p.

- zweimal täglich beobachten und dokumentieren [15]

- Kontrolle auch von Ikterus, Dehydratation und Allgemeinzustand

- Engmaschige Gewichtskontrollen, 1-mal täglich oder vor und nach jedem Stillen

- Ggf. Untersuchung der oralen Anatomie (z. B. kurzes Zungenbändchen)

- Erfassung mütterlicher Risikofaktoren für ein eingeschränktes Stillvermögen

\section{Beurteilung des Stillvorganges}

Ein Neugeborenes sollte in der Lage sein, alle 2-3 Std. (mind. 8-10-mal pro Tag) ca. 15 Min. lang effektiv zu saugen. Normalerweise wird es in diesem Rhythmus von selber zum Stillen wach. Zudem gibt es Phasen am Tag oder in der Nacht, in denen es über mehrere Stunden in sehr kurzen Abständen stillen möchte (Clusterfeeding).

Bei einem reifen Saugverhalten kommt es nach einer kurzen Stimulierungsphase mit rasch aufeinanderfolgenden, kurzen Saugbewegungen, zu kontinuierlichem Saugen und Schlucken mit langsamen, ausladenden Bewegungen des Unterkiefers. Erst nach mehrfachem Saugen und Schlucken von 10-30-Mal in Folge, einem sogenannten Saugschub, wird eine erste kleine Pause gemacht. Nach 5-7 Min. lässt die Dauer der Saugschübe allmählich nach und es werden häufiger kurze Pausen eingeschoben. Als Zeichen für einen guten Milchtransfer ist Schlucken nach jedem oder jedem 2. bis max. 3 . Saugvorgang zu beobachten. Während des gesamten Stillvorgangs, einschließlich der Pausen, wird der Saugschluss gehalten.

Ganz anders stellt sich das ineffektive Saugverhalten bei einem späten Frühgeborenen dar.

\section{Merkmale für Saugschwäche und unzureichendes Saugen}

- Kurze Saugschübe, lange Pausen

- Grübchenbildung in den Wangen

- Schmatzen durch häufiges Lösen des Saugschlusses

- Zittern des Unterkiefers in den Saugpausen

- Schlucken erst nach wiederholten Saugbewegungen

- Kurze Saugbewegungen

- Wenig langsame, ausladende Saugbewegungen

- Unzureichende Leerung der Brust

Es reicht also nicht aus, ein Kind 15 Min. lang an der Brust zu halten, vielmehr kommt es darauf an, sicherzustellen, dass es währenddessen effektiv saugt.

Späte Frühgeborene sind meistens nicht in der Lage, die Brust gut zu leeren, sodass sie selbst bei einer mehr als ausreichenden Milchmenge eine Zufütterung (vorzugsweise mit abgepumpter Muttermilch) benötigen. Ein reifes Saugverhalten wird manchmal erst mit 44 Gestationswochen erreicht, sodass die Milchbildung solange durch zusätzliches Abpumpen auf einem ausreichenden Niveau gehalten werden muss [12].

\section{Der Stillstart}

Wie für das reife Neugeborene gilt es für das späte Frühgeborene, das Bonding nach der Geburt zu ermöglichen. Das beinhaltet den ununterbrochenen Hautkontakt mit der Mutter (skin-to-skin) für mindestens 1 Std. nach der Geburt oder solange bis es gestillt wurde. Die Sorge um eine Belastung für das späte Frühgeborene ist unbegründet. Vielmehr konnte belegt werden, dass gerade bei ihm eine bessere kardio-respiratorische Stabilität zu erwarten ist. Auch wurden höhere Blutglukosewerte nach 75 und 90 Min. gemessen [19]. Bekanntermaßen unterstützt das Bonding nach der Geburt zudem eine stabile Körpertemperatur und die Aufnahme des ersten Stillens.

\section{Stillen sicherstellen}

Eine Trennung von Mutter und Kind, wie es zur besseren Beobachtung eines späten Frühgeborenen mitunter erfolgt, sollte möglichst vermieden werden. Rooming-in und viel Haut-an-Haut-Kontakt (Känguru-Methode) tragen im weiteren Verlauf zur Stabilisierung des kindlichen Allgemeinzustandes bei und begünstigen häufige Stillversuche.

Für Zuhause empfiehlt es sich ebenfalls, das Kind in Hör- und Sichtweite zu

\section{Ein schläfriges Kind wecken:}

- Wickeln oder Ausziehen

- Auf der Unterlage achsengerecht rollen

- Schlafpuppentechnik (mehrmals aufrichten und zurücklegen)

- Gezielt und bestimmt mit dem Kind reden

- Fußreflexzonenmassage

- Handinnenflächen massieren

- Rückenmassage

Auf Kältereize sollte verzichtet werden, weil die Gefahr der Auskühlung zu groß ist. Ein entkleidetes Kind kann aber rasch in Haut-an-Haut-Kontakt mit der Mutter gebracht werden, um dort allmählich wach zu werden. 


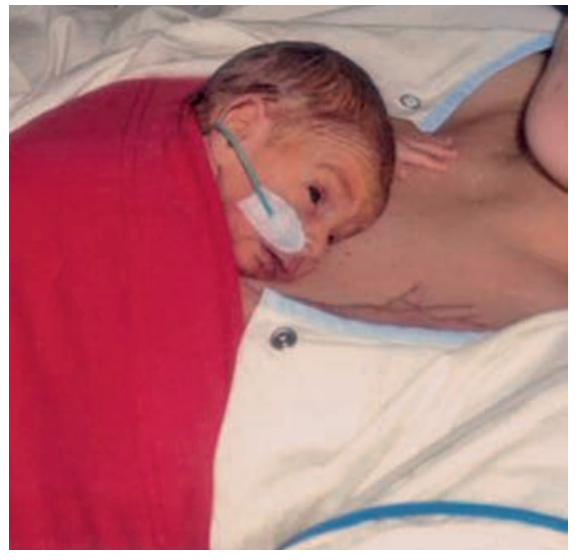

Abb. 1 Hautkontakt mit der Mutter. Aus: DHV (2012): Praxisbuch: Besondere Stillsituationen. Hippokrates Verlag Stuttgart.

haben. So kann die Mutter die ersten subtilen Stillzeichen wie Räkeln, Gähnen, leise Geräusche und Blinzeln wahrnehmen und unmittelbar darauf reagieren. Das Stillen sollte so mindestens alle 2-3 Std. erfolgen, anderenfalls muss das Kind in diesem Rhythmus zum Stillen geweckt werden.

\section{Ein wacher Säugling muss nicht noch} „wacher" gemacht werden. Er sollte vielmehr unverzüglich gestillt werden, um Energieverluste zu vermeiden!

\section{Nicht effektives Stillen}

Ist das Stillen nicht ausreichend effektiv, muss das weitere Vorgehen unverzüglich angepasst werden, um eine ausreichende Ernährung des Kindes sicherzustellen. Das beinhaltet:

- Häufig stillen und/oder füttern, d.h. (8-)10-12-Mal in 24 Std.

- Kurz stillen, nur solange wie effektives Saugen zu beobachten ist

- Stilldauer einschl. Zufüttern auf 20-30 Min. begrenzen [15]

- Mit der Milchgewinnung, dem Abpumpen beginnen

- Indikation für die Zufütterung großzügig stellen

Das Prinzip besteht in häufigen, kurzen und effektiven Mahlzeiten! Der Behandlungsplan stellt sicher, dass das Stillen fortgeführt werden kann und gleichzeitig ein ausreichender Aufbau der Milchbildung stattfindet.

Ein häufiger Behandlungsfehler besteht darin, späte Frühgeborene mit seltenen und entsprechend ausgedehnten Mahlzeiten zu versorgen. Sie sind dann so erschöpft, dass sie lange Schlafphasen entwickeln, nur schwer zu wecken und zu stillen sind und schließlich insgesamt zu wenig Nahrung bekommen. Außerdem entspricht dies nicht dem physiologischen Stillrhythmus, sodass sich der Übergang zum ausschließlichen Stillen umso schwieriger gestaltet.

In Teil 2 des Artikels in Heft 1/2015 beantwortet die Autorin die Frage, wie das Stillen gerade bei späten Frühgeborenen optimiert werden kann.

\section{Literatur}

1 Poets CF, Wallwiener D, Vetter K: Risks associated with delivering infants 2 to 6 weeks before term - a review of recent data. Dtsch Arztebl Int 2012; 109(43): 721-726

2 Leone A, Ersfeld P, Adams M, Schiffer PM, Bucher HU, Arlettaz R: Neonatal morbidity in singleton late preterm infants compared with full-term infants. Acta Paediatr. 2012 Jan; 101(1): 6-10

3 Gouyon et al.: Neonatal outcome associated with singleton birth at 34-41 weeks of gestation. International Journal of Epidemiology 2010; 39: 769-776

4 Cole S: Breastfeeding challenges made easy for late preterm infants: the go-to guide for nurses and lactation consultants. New York: Springer Publishing Company; 2014

5 Radtke JV: The Paradox of Breastfeeding-Associated Morbidity among Late Preterm Infants. J Obstet Gynecol Neonatal Nurs. 2011; 40(1): 9-24

6 Hallowell SG, Spatz DL: The Relationship of Brain Development and Breastfeeding in the Late-Preterm Infant. Journal of Pediatric Nursing 2012; 27: 154-162

7 Meier P, Patel AL, Wright K, Engstrom JL: Management of Breastfeeding During and After the Maternity Hospitalization for Late Preterm Infants. Clin Perinatol 2013; 40: 689-705

8 Walker M: Breastfeeding Management for the Late Preterm Infant: Practical Interventions for „Little Imposters“. Clinical Lactation 2010; Fall 1: 22-26

9 McDonald SW, Benzies KM, Gallant JE, McNeil DA, Dolan SM, Tough SC:

A Comparison Between Late Preterm and Term Infants on Breastfeeding and Maternal Mental Health. Matern Child Health J (2013) 17: 1468-1477

10 Ayton J, Hansen E, Quinn S, Nelson M: Factors associated with initiation and exclusive breastfeeding at hospital discharge: late preterm compared to 37 week gestation mother and infant cohort. International Breastfeeding Journal 2012; 7: 16

11 Radtke Demirci J, Sereika SM, Bogen D: Prevalence and Predictors of Early Breastfeeding Among Late Preterm Mother-Infants Dyads. Breastfeeding Medicine 2013; 8(3): 277-285

12 Meier PP, Furmann LM, Deghenhardt M: Increased Lactation Risk for Late Preterm Infants and Mothers: Evidence and Management Strategies to Protect Breastfeeding. J Midwifery Womens Health 2007; 52: 579-587

13 Deoni SCL, Dean DC, Piryatinsky I, O‘Muircheartaigh J, Waskiewicz N, Katie Lehman K, Han M, Dirks H: Breastfeeding and early white matter development: A cross-sectional study. NeuroImage 2013; 82: 77-86

14 Vice FL, Gewolb IH (2008). Respiratory patterns and strategies during feeding in preterm infants. Developmental Medicine \& Child Neurology 2008, 50 : 467-472

15 The Academy of Breastfeeding Medicine: Breastfeeding the Late Preterm Infant (34 0/7 to 36 6/7 Weeks Gestation). First Revision June 2011. Breastfeeding Medicine 2011; 3: 151-156

16 Radtke Demirci J, Happ MB, Bogen DL, Albrecht SA, Cohen SM: Weighing worth against uncertain work: The interplay of exhaustion, ambiguity, hope and disappointment in mothers breastfeeding late preterm infants. Matern Child Nutr. Author manuscript; available in PMC 2014 April 01

17 Ludwig SM: Oral Feeding and the Late Preterm Infant. In: Newborn \& Infant Nursing Rewiews, Volume 7, Issue 2, Pages 72-75; June 2007 
18 Zanardo V, Gambina I, Begely C, Cosmi E, Giustardi A, Trevisanuto D: Psychological distress and early lactation performance in mothers of late preterm infants. Early Human Development 2011; 87: 321-323

19 Moore ER, Anderson GC, Bergman N, Dowswell T. Early skin-to-skin contact for mothers and their healthy newborn infants. Cochrane Database of Systematic Reviews 2012, Issue 5
20 Tsang RC, Uauy R, Koletzko B, Zlotkin SH, Hrsg.: Nutrition of the Preterm Infant: Scientific Basis and Practical Guidelines. (C) Digital Educational Publishing, Inc.; Provided Courtesy of Mead Johnson Nutritionals, 2005

21 Thomas J, Marinelli KA, Hennessy M, and the Academy of Breastfeeding Medicine Protocol Committee: ABM Clinical Protocol \#16: Breastfeeding the Hypotonic Infant. Breastfeeding Medicine, Volume 2, Number 2, 2007

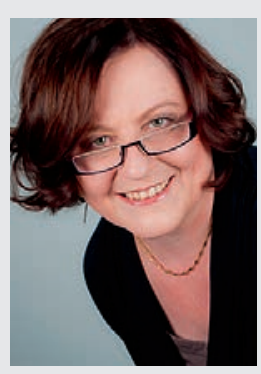

Hella R. Köster

Brüderstr. 15

42719 Solingen info@maieutika.de 